\title{
A miniaturised light-sheet microscopy system using MEMS micromirror control
}

\author{
Spyridon Bakas ${ }^{1}$, Deepak Uttamchandani ${ }^{1}$, Hiroshi Toshiyoshi ${ }^{2}$, and Ralf Bauer ${ }^{1}$ \\ ${ }^{1}$ University of Strathclyde, Glasgow, United Kingdom \\ ${ }^{2}$ University of Tokyo, Tokyo, Japan \\ spyridon.bakas@strath.ac.uk
}

\begin{abstract}
We present the optical characterization of a MEMS enabled light-sheet microscopy system which combines 2-axis MEMS control over the light delivery for a scanned and freely positioned light-sheet as well as MEMS focal control of the image collection. The system performance is evaluated using standard imaging targets and fluorescence bead samples for 3D image generation.
\end{abstract}

Keywords-MEMS micromirror, light-sheet microscopy, miniaturized biomedical imaging system, fluorescence imaging

\section{INTRODUCTION}

The field of microscopy in biomedical sciences is steadily moving towards solutions that aim to provide high speed, high resolution 3D imaging with minimal disturbance and damage to the sample. A critical amount of these requirements are met by light-sheet microscopy (LSM) [1] which has proven to provide images of cell scale samples with reduced light doses, thereby minimizing undesired effects such as photobleaching. Even though research in LSM is rapidly growing [2], such system usually come at a significant financial cost. Our research focuses on using MEMS as the active elements in LSM, reducing the cost and size of the system by providing external dynamic control in the imaging process. The approach of using MEMS in light delivery and collection for LSM systems has been previously shown in [3] and [4]. In this paper we will present the incorporation of two MEMS in a miniaturised LSM, which allows imaging of different planes of fluorescent test samples through external control and optical path synchronization.

\section{LSM DESIGN AND CHARACTERISTICS}

\section{A. Laser light-sheet path}

The illumination path of the LSM (Fig.1 (a)) is designed in order to create a thin planar light-sheet, with the capability of digital control over the light-sheet position. The active element to accomplish this purpose is a 2-axis piezoelectric MEMS micromirror [4][5] (Fig. 1(b)). The $1 \mathrm{~mm}$ diameter, aluminium coated, mirror reflects the collimated laser beam (CNI $473 \mathrm{~nm}$ solid state laser) at $45^{\circ}$ incidence onto a $4 \mathrm{f}$ lens system consisting of a $\mathrm{f}=7.5 \mathrm{~mm}$ and $\mathrm{f}=10 \mathrm{~mm}$ achromatic lens. A telecentric setup is completed with an additional $\mathrm{f}=7.5 \mathrm{~mm}$ lens, leading to a $5.2 \mathrm{~mm}$ working distance. The propagation length between the MEMS and the focal plane adds up to $1=80 \mathrm{~mm}$. The MEMS consists of static spiral shaped PZT actuators on the outside and an inner ring resonant PZT actuator. The static actuators are responsible for positioning the laser beam along the $\mathrm{x}$-axis, whereas the resonant movement of the inner actuator at $\mathrm{f}=37.4 \mathrm{kHz}$ creates the scanned light-sheet on the vertical y-axis. The static rotation of the mirror is controlled through an Arduino Due board whereas the resonance movement is achieved with a signal generator.

This work was supported by the Royal Academy of Engineering under the Engineering for Development Research Fellowship scheme (RF1516 15\8).

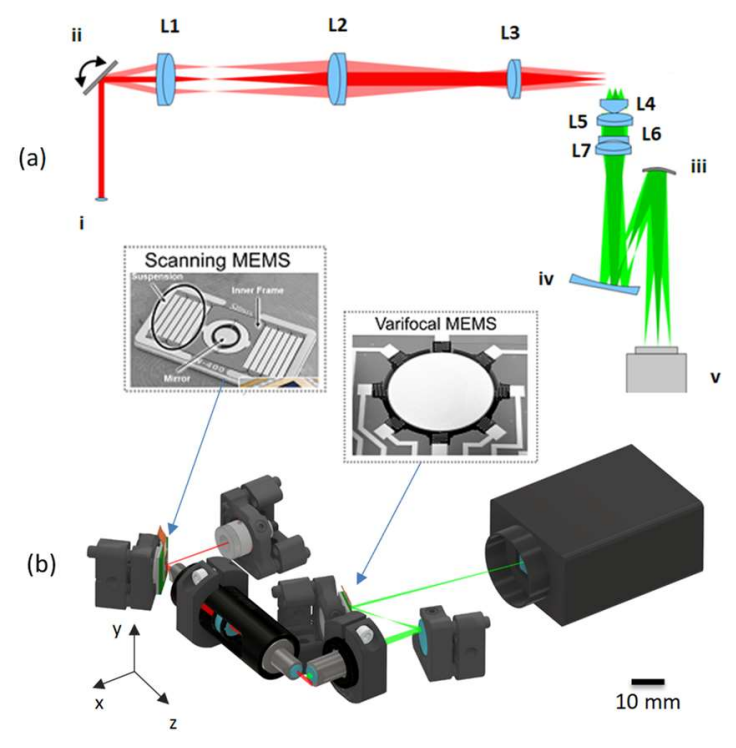

Fig. 1. (a) Optical paths of miniaturized LSM. Laser beam propagation path (red), from left to right: $\mathrm{i}$ - CNI 473nm solid state laser source, ii - PZT mirror, group of lenses (L1, L2, L3). Imaging path (green) from top to bottom: group of lenses (L4, L5, L6, L7), iii - varifocal bimorph MEMS, iv - concave silver mirror ROC $=38 \mathrm{~mm}, \mathrm{v}-$ FLIR Grasshopper sCMOS camera. (b) 3D schematic for miniaturized LSM including SEM images of the scanning and varifocal MEMS. The image illustrates 3D printed lens adaptors used for incorporating the lens groups in the Thorlabs $1 / 2$ " lens tubes, making the system more robust and minimizing stray light.

The measured full width half maximum (FWHM) beam diameter along the propagation axis, $z$, is shown in Fig 2 (a). The beam characteristics were measured with a knife edge test using a Thorlabs MTS25-Z8 motorized stage with step size of $0.7 \mu \mathrm{m}$. The focal spot size was found to be $2.85 \mu \mathrm{m}$. The diameter determines the thickness of the light-sheet, which remains below $4.5 \mu \mathrm{m}$ in the imaging field of view (FOV) of $210 \mu \mathrm{m}$. The light propagation was additionally measured with an orthogonal static fluorescence imaging system using a x10 objective (Fig. 2(b)), showing the fluorescence response in the focal region in a cuvette filled with fluorescein sodium salt (SIGMA-ALDRICH, F6377) diluted in water. The measured power of the laser in the focal plane area is $2.6 \mathrm{~mW}$.

\section{B. Imaging path}

The imaging path of the miniaturized LSM system (Fig. $1(\mathrm{a}))$ is designed to provide image resolution $<1 \mu \mathrm{m}$ as well as variable focus. In order to achieve this two groups of lenses are used, followed by a $1.8 \mathrm{~mm}$ diameter varifocal thermal bimorph MEMS micromirror that reflects the image towards the FLIR Grasshopper sCMOS camera (GS3-U3-32S4M-C). A $500 \mathrm{~nm}$ thick gold layer is deposited on top of the $10 \mu \mathrm{m}$ thick silicon device to enable thermal bimorph actuation [4]. A $500 \mathrm{~nm}$ long-pass filter (Thorlabs FELH0500) was used during fluorescence imaging to block all excitation light. The thermal actuation of the varifocal MEMS is used to change the Radius of Curvature (ROC) of the mirror surface between 

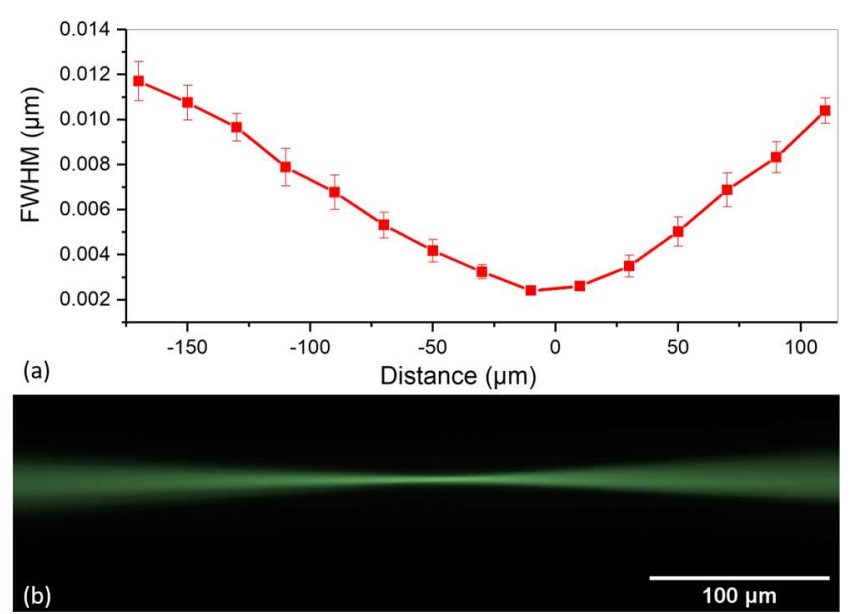

Fig. 2. (a) Light sheet excitation beam propagation characteristics through the focal plane area. (b) Light sheet propagation imaged through a cuvette containing fluorescein solution. The image is captured using a test imaging setup $(\mathrm{x} 10, \mathrm{NA}=0.25)$ orthogonal to the propagation path.

$32 \mathrm{~mm}$ and $60 \mathrm{~mm}$, translating in this way the optical path imaging focal plane by $120 \mu \mathrm{m}$. The static and dynamic actuation of the mirror is controlled with the Arduino Due.

Characterization of the imaging path is accomplished with the use of a 1951 USAF negative target. The target is positioned in the focal plane intersection of the excitation and imaging path at an angled position $\left(\theta \simeq 30^{\circ}\right)$ similar to the microscope slide positioning of samples (Fig. 3 (a)). The system was aligned to ensure overlap of the beam waist of the laser beam in the centre of the imaging FOV with a working distance of the imaging path of $2 \mathrm{~mm}$.

As is presented in Fig. 3 (b) and Fig 3. (c), the imaging system is able to resolve the smallest group 7-6 of the target, achieving a resolution well below $2.2 \mu \mathrm{m}$. The out-of-focus appearance of the higher group numbers due to the angled imaging target is also shown in Fig. 3 (b).

\section{FLUORESCENCE IMAGING METHOD AND RESULTS}

To demonstrate and evaluate fluorescence imaging a FocalCheck $^{\mathrm{TM}}$ Fluorescence Microscope Test Slide (ThermoFischer Scientific, F36909) was used. $15 \mu \mathrm{m}$ diameter fluorescent coated beads fixed in optical cement are used for 3D image demonstration. The experimental method for imaging requires the test slide to be placed in the same position of the imaging target. The position accomplishes an additional purpose as the angled microscope slide gives the opportunity of addressing various fixed microbeads at different focal planes of the microscope slide towards the $\mathrm{x}$ axis. Synchronised positioning of the two MEMS mirrors in

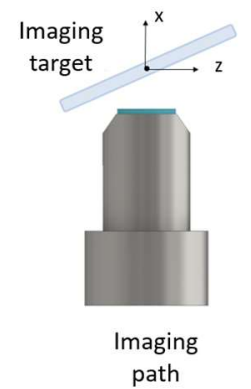

(a)

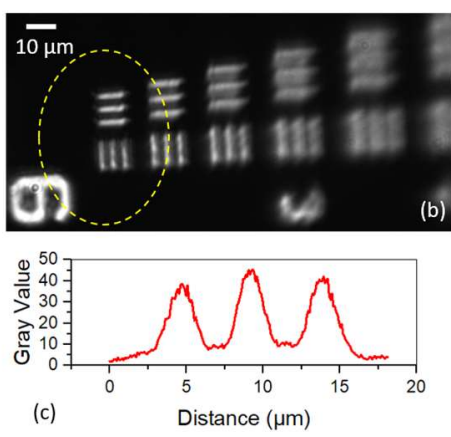

Fig. 3. (a) 3D schematic top view of target positioning towards the imaging path. (b) Image of group 7 of 1951 USAF target (highlighted area is in focus) (c) Imaging group 7-6 cross section profile.
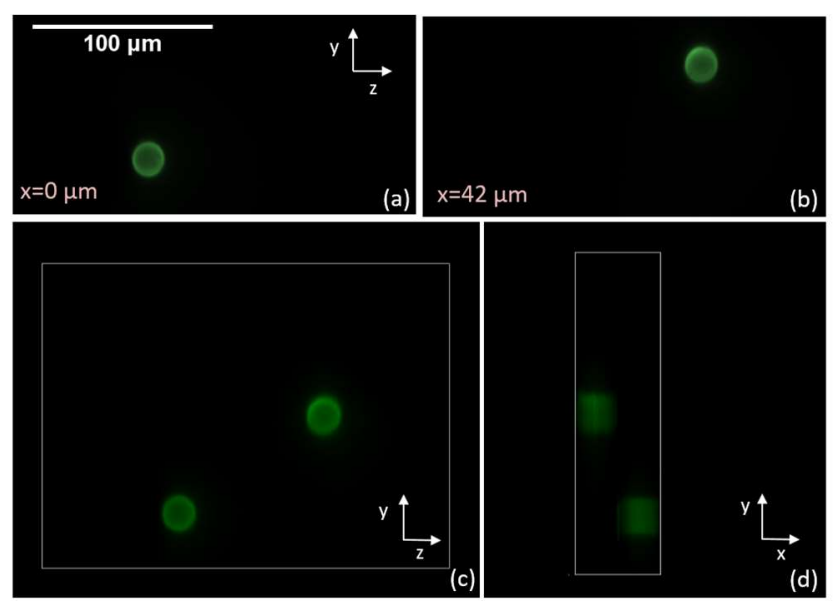

Fig. 4. $15 \mu \mathrm{m}$ fluorescent beads imaged in 3D; (a) Image slice at focal plane at $\mathrm{x}=0 \mu \mathrm{m}$ (b) Image slice at focal plane at $\mathrm{x}=42 \mu \mathrm{m}$ (c) $3 \mathrm{D}$ reconstruction of the sample in $x / y$ and (d) in $y / z$.

the two optical paths allows for accurate control of the lightsheet position and imaging. The first focal plane in Fig 4 (a) is illuminated with the laser scan line positioned at $\mathrm{x}=0 \mu \mathrm{m}$ and with a light sheet height of $200 \mu \mathrm{m}$. A camera exposure time of $4.8 \mathrm{~ms}$ and gain of $11 \mathrm{~dB}$ was used for image collection. By varying the static angle of the scanning MEMS mirror the light-sheet gets translated to $\mathrm{x}=42 \mu \mathrm{m}$ to image a second bead in the FOV. Accordingly, the varifocal MEMS is actuated to transfer the focal plane to the same position through the mirror ROC change. Synchronised actuation in steps of $x=3.5 \mu \mathrm{m}$ allows sectioning of the individual beads and $3 \mathrm{D}$ reconstruction. The results presented in Fig. 4 (c) and Fig 4 (d) show a down sampled reconstructed 3D capture formed by 30 image planes.

\section{CONCLUSION}

The optical design and characterisation of a miniaturized light-sheet microscope is presented, using a piezoelectrically actuated scanning mirror in the laser beam propagation path and a electrothermally actuated varifocal mirror in the imaging path for 3D imaging without sample movement. The two paths are characterized individually in terms of the beam propagation profile and imaging resolution respectively. It is shown that the synchronization of the two mirrors can address multiple focal planes of the sample as well as provide sectioning of individual objects in the FOV. Three dimensional fluorescent images of a $15 \mu \mathrm{m}$ bead target are presented and constitute the first step towards 3D light-sheet microscopy fully controlled by MEMS.

\section{REFERENCES}

[1] R. M. Power and J. Huisken, "A guide to light-sheet fluorescence microscopy for multiscale imaging," Nat. Methods, vol. 14, no. 4, pp. 360-373, 2017.

[2] O. E. Olarte, J. Andilla, E. Gualda and P. Loza-Alvarez, "Light-sheet microscopy: A tutorial," Adv. Opt. Photonics, vol. 10, no.1, pp. 111179, 2018.

[3] R. Bauer and D. Uttamchandani, "MEMS micromirror based light sheet generator for biomedical imaging," in 2017 International Conference on Optical MEMS and Nanophotonics (OMN), 2017, pp. 129-130.

[4] S.Bakas, D. Uttamchandani, H. Toshiyoshi and R. Bauer "MEMS enabled control of light-sheet microscopy optical beam paths", in 2018 International conference on Optical MEMS and Nanophotonics (OMN), 2018, pp. 145-146.

[5] S. Jeon and H. Toshiyoshi, "MEMS tracking mirror system for a bidirectional free-space optical link," Appl. Opt., vol. 56, no. 24, pp. $6720-6727,2017$. 